\title{
High Age Increases the Risk of Complications and Reduces Survival Following Esophagectomy for Adenocarcinoma
}

\author{
Daniel Willy Kjaer ${ }^{1,}$, Kaare Terp Fjerderholt ${ }^{1}$, Jakob Kirkegaard ${ }^{1}$, Cecilie Okholm ${ }^{2}$, \\ Michael Patrick Achiam², Lars Bo Svendsen ${ }^{2}$, Frank Viborg Mortensen ${ }^{1}$ \\ ${ }^{1}$ Department of Surgery, Aarhus University Hospital, Aarhus, Denmark \\ ${ }^{2}$ Department of Surgical Gastroenterology, Rigshospitalet, Copenhagen, Denmark
}

Email address:

Dankja@rm.dk (D. W. Kjaer)

${ }^{*}$ Corresponding author

\section{To cite this article:}

Daniel Willy Kjaer, Kaare Terp Fjerderholt, Jakob Kirkegaard, Cecilie Okholm, Michael Patrick Achiam, Lars Bo Svendsen, Frank Viborg Mortensen. High Age Increases the Risk of Complications and Reduces Survival Following Esophagectomy for Adenocarcinoma. Journal of Surgery. Vol. 6, No. 1, 2018, pp. 1-7. doi: 10.11648/j.js.20180601.11

Received: November 8, 2017; Accepted: November 16, 2017; Published: December 14, 2017

\begin{abstract}
Introduction: The aim of the present study was to investigate the impact of high age and comorbidities in relation to postoperative complications, mortality, and long-term survival in patients undergoing surgery for adenocarcinoma of the esophagus and gastro-esophageal junction (GEJ). Methods: A cohort study of 557 patients operated for adenocarcinoma of the esophagus and GEJ. Data were collected from a prospectively maintained database, the Danish National Patient Registry, and medical records. Univariate and multivariate statistical models were used to analyze data after stratification for possible confounders. Results: The incidence of postoperative complications increased in patients aged $\geq 75$ years compared with younger patients (OR: 1.57; 95\% CI: 0.93-2.62) although not significant. However, testing for trend revealed a linear increase with age for severe complications $(\mathrm{p}=0.005)$. Age $\geq 75$ years was also associated with increased 90-day but not 30-day mortality (HR: 5.05; 95\% CI: 1.70-14.94 and HR: 3.47; 95\% CI: 0.61-19.72 respectively). Overall survival decreased with increasing age $(\mathrm{p}=0.036)$. Conclusion: We found high age as an independent risk factor for death within 90 days but not 30 days after surgery for adenocarcinoma of the esophagus and GEJ. The presence of co-morbidity seemed to increase the risk for postoperative complications.
\end{abstract}

Keywords: Esophageal Neoplasms, Adenocarcinoma, Aged, Esophagectomy

\section{Introduction}

The incidence of esophageal cancer and gastroesophageal junction (GEJ) cancer is rising, rendering it the sixth most common cause of cancer-related death worldwide [1]. Despite the recent introduction of multimodal regimes, surgery is still the preferred curative treatment option for esophageal and GEJ cancer [2]. However, resection of esophageal and GEJ cancer is a major procedure and it is therefore mandatory to ensure that potential patients present with an acceptable performance status without severe comorbidities.

With the increase in average life expectancy in the general population, more and more people in their 70's and 80's are referred for evaluation due to the occurrence of esophageal and GEJ cancer. Many of these patients have resectable tumors and are thus referred to surgery. However, the incidence of comorbidities, is known to increase with age and performance, in general, is known to decrease with age. In the elderly patients in particular, the risk-assessment prior to esophageal surgery is therefore of paramount importance.

The aim of this study was to investigate the importance of high age and comorbidities in relation to postoperative complications, 30- and 90-day mortality, and survival following surgery for adenocarcinoma of the esophagus and GEJ.

\section{Materials and Methods}

\subsection{Study Population}

Using the Danish Registry of Esophageal, 
Gastroesophageal junction (GEJ), and Gastric cancers (the $D E C V$ database), we assembled our cohort by identifying all patients undergoing curative intended surgery for esophageal and GEJ cancer at two Danish institutions during the period January 1, 2003 to December 31, 2012. Established in 2003, the DECV database is a nationwide and prospectively maintained national registry, including all cancers of the esophagus, GEJ, and stomach in Denmark. After initial identification of 801 patients, we included patients with adenocarcinoma $(n=613)$ and, from this subset of patients, individuals who had undergone esophageal resection with gastric pull up ( $\mathrm{n}=558)$. One patient had been misclassified and had not undergone surgery. Our final study population entailed 557 consecutive patients. Patients were followed from the day of surgery to the day of death or end of followup (August 10, 2015), whichever occurred first.

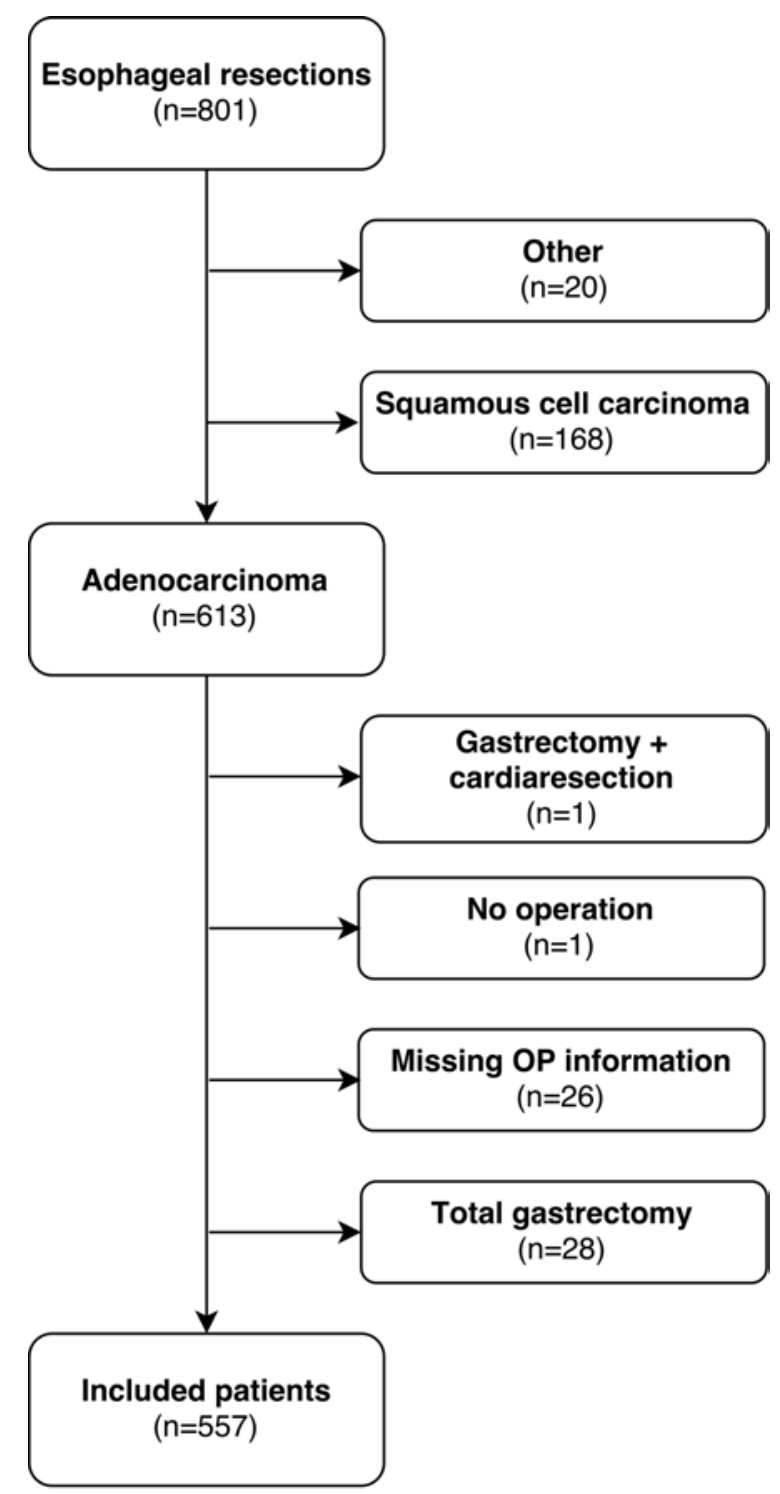

Figure 1. Flowchart of the study population.

\subsection{Information on Patients and Procedures}

We used the patients' unique CPR number, which is an identification number assigned to all Danish residents at birth or immigration allowing individual-level cross-linkage between several Danish medical registries, to identify the patients' medical records for further review. We collected information on age, sex, smoking, histology, type and date of operation, vital status and date of death, pTNM, comorbidity, use of neoadjuvant chemoradiotherapy, and complications including anastomotic leakage. Anastomotic leakage was defined as radiologically or endoscopically recognized leaks from the area surrounding the anastomosis.

We divided patients into three age categories $(<70$ years, $\geq$ 70 - $<75$ years, and $\geq 75$ years). Complications were defined according to the Clavien-Dindo classification [3] as suggested by the international consensus report on complications associated with esophagectomy from 2015 [4]. When multiple complications or interventions were present, only the one deemed most severe was included in the analysis. Complications were further divided into a dichotomous variable (yes/no), which was used for analytical purposes.

The use of NSAID within the first seven postoperative days was recorded. All patients underwent surgery ad modum Ivor-Lewis with or without modification by minimal invasive surgery [5].

\subsection{Data on Comorbidity}

We assessed the patients' comorbid burden using the Charlson Comorbidity Index (CCI) [6]. In the CCI, which is a validated, rated scoring system of comorbidities, each diagnosis is assigned a score based on the risk of 1-year mortality. We grouped the CCI score into three categories: 0 (low comorbidity), 1 (moderate comorbidity), and $\geq 2$ (high comorbidity). This subdivision for CCI is common practice in advanced surgery, since patients with severe comorbidity do not undergo high risk procedures such as esophageal resection with gastric pull up [7]. Information on the diagnoses included in the CCI was retrieved from the Danish National Patient Registry (DNPR). The DNPR is a nationwide registry that covers every hospital admission in Denmark since 1977. The DNPR include information on dates of admission and discharges, surgical procedures, and discharge diagnoses. Since 1994, diagnoses in the DNPR have been classified according to the $10^{\text {th }}$ edition of the International Classification of Diseases (ICD-10). Identification of the patients in the DNPR was obtainable using the CPR number. This study was approved by the Danish Data Protection Agency (J. nr. 2007-58-0010 and J. $n r$. 1-16-02-197-15) and the National Board of Health.

\subsection{Statistical Analyses}

Clinical and pathological patient characteristics are presented as distribution frequencies (numbers and percentages) within each of the three age groups. Continuous variables are listed as means, if normally distributed, along with the corresponding $95 \%$ confidence interval (CI). Categorical data were analyzed using $\chi^{2}$-test; in case of 
insufficient numbers in the contingency tables, Fishers exact test was used. Ordinal and binomial outcome variables were tested using non-parametric test for trends. Unknown information of smoking status was accounted for by multiple imputation. First, the prevalence and pattern of missing prognostic covariates were assessed and deemed not to be missing completely at random. On this background, multiple imputations $(n=20)$ were performed $[8,9]$. Multivariate analyses were performed using binomial logistic regressions for complications and the Cox proportional hazards regression model for 30-day and 90-day mortality. Survival was presented using Kaplan-Meier plots, and age groups were compared using the log-rank test. In multivariate analyses, we adjusted for age, gender, smoking, CCI score, NSAID use, and type of operation. P-values $<0.05$ were considered statistically significant. Statistical analyses were performed using STATA ${ }^{\circledR} 13.1$ (StataCorp LP, College Station, Texas, USA).

\section{Results}

\subsection{Demographics}

Of the 557 patients included in this study, $468(84.0 \%)$ were male (Table 1). The mean age at surgery was 64.1 (range: 28.2-85.4) years with an overweight of female patients in the oldest age group compared with the younger age groups, although this was not significant. In total, 188 patients $(45.2 \%)$ were current smokers, and more smokers than ex-smokers or non-smokers $(\mathrm{p}=0.000)$ were found among the younger patients compared to the older patients. Three hundred fifty-nine patients $(64.5 \%)$ had a comorbidity score of $\geq 2$ according to the CCI. There was a significant difference in the general distribution of comorbidity in the three age groups $(\mathrm{p}=0.019)$, but the trend was not monotonic $(\mathrm{p}=0.367)$. The vast majority of the tumors were T2-T3/N0N1, which accounted for approximately $80 \%$. Only 131 $(23.5 \%)$ of the patients received neoadjuvant treatment, as it was not a standard treatment prior to 2010 [10].

Table 1. Characteristics of 557 patients undergoing resection for adenocarcinoma of the esophagus and gastroesophageal junction.

\begin{tabular}{|c|c|c|c|c|c|}
\hline & $<70$ years $(\%)$ & $\geq 70-<75$ years $(\%)$ & $\geq 75$ years $(\%)$ & Total (\%) & $\mathbf{P}$ \\
\hline Total & 398 & 78 & 81 & 557 & \\
\hline Gender & & & & & 0.123 \\
\hline Female & $60(15.1)$ & $10(12.8)$ & $19(23.5)$ & $89(16.0)$ & \\
\hline Male & $338(84.9)$ & $68(87.2)$ & $62(76.5)$ & $468(84.0)$ & \\
\hline Smoking & & & & & 0.000 \\
\hline Yes & $147(50.2)$ & $21(36.2)$ & $20(30.8)$ & $188(45.2)$ & \\
\hline No & $73(24.9)$ & $9(15.5)$ & $12(18.5)$ & $94(22.6)$ & \\
\hline Missing values & 105 & 20 & 16 & 141 & \\
\hline T-stage & & & & & 0.490 \\
\hline T0 & $13(3.3)$ & $2(2.6)$ & $1(1.2)$ & $16(2.9)$ & \\
\hline $\mathrm{T} 1$ & $54(13.6)$ & $16(20.5)$ & $8(9.9)$ & $78(14.0)$ & \\
\hline $\mathrm{T} 2$ & $155(38.9)$ & $31(39.7)$ & $37(45.7)$ & $223(40.0)$ & \\
\hline $\mathrm{T} 3$ & $166(41.7)$ & $29(37.2)$ & $33(40.7)$ & $228(40.9)$ & \\
\hline $\mathrm{T} 4$ & $10(2.5)$ & 0 & $2(2.5)$ & $12(2.2)$ & \\
\hline N-stage & & & & & 0.136 \\
\hline N1 & $174(43.7)$ & $43(55.1)$ & $30(37.0)$ & $247(44.3)$ & \\
\hline N2 & $59(14.8)$ & $10(12.8)$ & $11(13.6)$ & $80(14.4)$ & \\
\hline N3 & $27(6.8)$ & $1(1.3)$ & $9(11.1)$ & $37(6.6)$ & \\
\hline CCI score & & & & & 0.019 \\
\hline 0 & $107(26.9)$ & $25(32.1)$ & $20(24.7)$ & $152(27.3)$ & \\
\hline 1 & $29(7.3)$ & $3(3.9)$ & $14(17.3)$ & $46(8.3)$ & \\
\hline$\geq 2$ & $263(65.8)$ & $50(64.1)$ & $47(58.0)$ & $360(64.5)$ & \\
\hline Neoadjuvant treatment & & & & & 0.982 \\
\hline No & $305(76.6)$ & $59(75.6)$ & $62(76.5)$ & $426(76.5)$ & \\
\hline Yes & $93(23.3)$ & $19(24.4)$ & $19(23.5)$ & $131(23.5)$ & \\
\hline Operation & & & & & 0.000 \\
\hline Laparotomy + Thoracotomy & $341(86.1)$ & $66(84.6)$ & $70(86.4)$ & $478(86.0)$ & \\
\hline Laparoscopy + Thoracotomy & $53(13.4)$ & $12(15.4)$ & $6(7.4)$ & $71(12.8)$ & \\
\hline Laparoscopy + Thoracoscopy & $2(0.5)$ & 0 & $5(6.2)$ & $7(1.3)$ & \\
\hline Missing values & 2 & 0 & 0 & 2 & \\
\hline No & $351(92.1)$ & $69(92.0)$ & $79(93.8)$ & $496(92.4)$ & \\
\hline Missing values & 17 & 3 & 0 & 20 & \\
\hline NSAID & & & & & 0.479 \\
\hline Yes & 79 (19.9) & $11(14.3)$ & $17(21.0)$ & $107(19.2)$ & \\
\hline No & $319(80.2)$ & $66(85.7)$ & $64(79.0)$ & $449(80.8)$ & \\
\hline Missing values & 0 & 1 & 0 & 1 & \\
\hline
\end{tabular}

CCI: Charlson Comorbidity Index; NSAID: Non-steroidal anti-inflammatory drug. 


\subsection{Complications}

The incidence of postoperative complications increased in patients aged $\geq 75$ years compared with younger patients (OR: $1.57 ; 95 \%$ CI: $0.93-2.62$ ), even though there were no general significant association in the distribution of complications within the three age groups $(\mathrm{p}=0.070)$ testing for trend revealed a monotonic linear increase indicating that patients in the group above 75 years had more severe complications $(\mathrm{p}=0.005)$. Testing age as a continuous variable with regard to postoperative complications was used to test the statistical significance of the result (OR: $1.02 ; 95 \%$ CI: 1.004-1.043). The risk of complications was slightly, although insignificantly, elevated in patients with comorbidity, defined as CCI score $>0$ (Table 2).

Table 2. Complications in 557 patients undergoing resection for adenocarcinoma of the esophagus and gastroesophageal junction.

\begin{tabular}{|c|c|c|c|c|c|}
\hline \multirow{2}{*}{ Total } & $<70$ years $(\%)$ & $\geq 70-<75$ years $(\%)$ & $\geq 75$ years $(\%)$ & Total (\%) & \multirow{2}{*}{$\mathbf{P}$} \\
\hline & 398 & 78 & 81 & 557 & \\
\hline Clavien-Dindo & & & & & 0.070 \\
\hline No complications & $218(54.8)$ & $37(47.4)$ & $35(43.2)$ & $290(52.1)$ & \\
\hline I & $14(3.5)$ & $1(1.3)$ & 0 & $15(2.7)$ & \\
\hline II & $91(22.7)$ & $18(23.1)$ & $22(27.2)$ & $131(23.5)$ & \\
\hline IIIa & $27(6.8)$ & $6(7.7)$ & $4(4.9)$ & $37(6.6)$ & \\
\hline IIIb & $21(5.3)$ & $7(9.0)$ & $6(7.4)$ & $34(6.1)$ & \\
\hline $\mathrm{IVb}$ & $6(1.5)$ & $3(3.9)$ & $3(3.7)$ & $11(2.0)$ & \\
\hline $\mathrm{V}$ & $3(0.8)$ & $1(1.3)$ & $4(4.9)$ & $8(1.4)$ & \\
\hline 30-day mortality & & & & & 0.015 \\
\hline Alive & $395(99.3)$ & $77(98.7)$ & $77(95.1)$ & $548(98.4)$ & \\
\hline Dead & $3(0.7)$ & $1(1.3)$ & $4(4.9)$ & $8(1.4)$ & \\
\hline 90-day mortality & & & & & 0.000 \\
\hline Alive & $391(98.2)$ & $75(96.2)$ & $72(88.9)$ & $538(96.6)$ & \\
\hline
\end{tabular}

Anastomotic leakage was identified in $7.6 \%(\mathrm{p}=0.646)$ of the patients, pulmonary and wound complications were present in $27.3 \%(\mathrm{p}=0.948)$ and $6.3 \%(0.985)$ of the patients, respectively, with no significant difference between the age groups. The only group of complications with a monotonic trend demonstrating increased incidence with increase age was the group of cardiac complications, present in $15.8 \%$ of the patients $(\mathrm{p}<0.001)$. Even though there was no difference in the distribution of patients treated with NSAID in the first seven days postoperatively, the adjusted logistic regression model showed a significant influence of NSAID in relation to complications (Table 3). Type of surgery (laparoscopy + thoracotomy) was associated with an increased occurrence of complications (OR: 2.35; 95\% CI: 1.37-4.02). Neoadjuvant treatment was associated with fewer postoperative complications (OR: $0.65 ; 95 \%$ CI: 0.44-0.97).

Table 3. Multivariate analyses of complications and mortality in 557 patients undergoing resection for adenocarcinoma of the esophagus and gastroesophageal junction.

\begin{tabular}{|c|c|c|c|c|c|c|}
\hline & \multirow{2}{*}{$\begin{array}{l}\text { Complications } \\
\text { OR (95\% CI) }\end{array}$} & \multirow{2}{*}{$\mathbf{P}$} & \multirow{2}{*}{$\begin{array}{l}\text { 30-day mortality } \\
\text { HR }(95 \% \text { CI })\end{array}$} & \multirow{2}{*}{$\mathbf{P}$} & 90-day mortality & \multirow{2}{*}{$\mathbf{P}$} \\
\hline & & & & & HR $(95 \%$ CI $)$ & \\
\hline \multicolumn{7}{|l|}{ Age } \\
\hline$<70$ & 1.00 (ref) & & 1.00 (ref) & & 1.00 (ref) & \\
\hline$\geq 70-<75$ & $1.37(0.83-2.29)$ & 0.218 & $1.44(0.14-14.69)$ & 0.755 & $2.17(0.52-8.75)$ & 0.276 \\
\hline$\geq 75$ & $1.57(0.93-2.62)$ & 0.089 & $3.47(0.61-19.72)$ & 0.160 & $5.05(1.70-14.94)$ & 0.003 \\
\hline \multicolumn{7}{|l|}{ Gender } \\
\hline Male & $0.77(0.48-1.24)$ & 0.282 & $0.25(0.05-1.17)$ & 0.078 & $0.44(0.16-1.23)$ & 0.118 \\
\hline Smoking & $1.03(0.81-1.30)$ & 0.825 & $1.72(0.48-6.02)$ & 0.403 & $1.31(0.67-2.54)$ & 0.426 \\
\hline \multicolumn{7}{|l|}{ CCI score } \\
\hline 0 & 1.00 (ref) & - & 1.00 (ref) & - & 1.00 (ref) & - \\
\hline 1 & $1.89(0.94-3.82)$ & 0.074 & $2.97(0.17-51.90)$ & 0.455 & $1.10(0.20-6.06)$ & 0.911 \\
\hline$\geq 2$ & $1.31(0.88-1.94)$ & 0.184 & $2.26(0.25-20.15)$ & 0.466 & $0.77(0.26-2.29)$ & 0.638 \\
\hline Yes & $1.91(1.23-2.96)$ & 0.004 & $0.69(0.08-5.90)$ & 0.733 & $2.23(0.82-6.06)$ & 0.118 \\
\hline \multicolumn{7}{|l|}{ Type of surgery } \\
\hline Open & 1.00 (ref) & - & 1.00 (ref) & - & 1.00 (ref) & - \\
\hline Laparoscopy + Thoracotomy & $2.35(1.37-4.02)$ & 0.002 & $0.83(0.09-7.58)$ & 0.866 & $1.87(0.56-6.04)$ & 0.295 \\
\hline Laparoscopy + thoracoscopy & $1.03(0.21-5.09)$ & 0.967 & 0.00 & 1.000 & 0.00 & 1.000 \\
\hline
\end{tabular}

OR: Odds ratio; HR: Hazard ratio; CI: Confidence interval; CCI: Charlson Comorbidity Index. 


\subsection{0- and 90-day Mortality}

Age $\geq 75$ years was associated with increased 90-day, but not 30-day, mortality (HR: 5.05 ; $95 \% \mathrm{CI}$ : $1.70-14.94$ and HR: 3.47; 95\% CI: $0.61-19.72$, respectively). None of the 41 patients with anastomotic leakage died within 30 days of surgery but there was a statistically significant increase in mortality with $12.2 \%$ of these patients dead at 90 days postoperatively compared to $3.0 \%$ in the group of patients without anastomotic leakage $(\mathrm{p}=0.003)$.

\subsection{Long-Term Survival}

The five-year survival rate was $25.1 \%$ as illustrated in the Kaplan-Meier plot (Figure 2). The overall survival curves for the three age groups differs significantly $(\mathrm{p}=0.036)$.

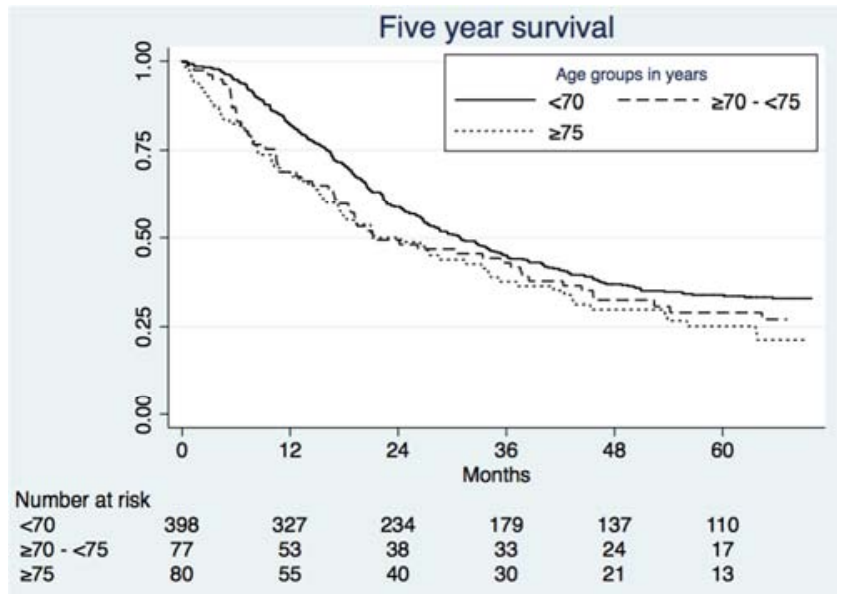

Figure 2. Survival curves of 557 patients undergoing resection for adenocarcinoma of the esophagus and gastroesophageal junction.

\section{Discussion}

In the present cohort study of 557 consecutive patients undergoing surgery for adenocarcinoma of the esophagus or GEJ, we investigated the risk of complications in addition to 30- and 90 days-mortality with special emphasis on age and comorbidities. All patients underwent esophagectomy with gastric pull-up ad modum Ivor-Lewis. Age $\geq 75$ years seemed to be a predictor for postoperative complications in addition to increased 90-day, but not 30-day, mortality. Presence of comorbidity may increase the risk of complications but does not affect mortality. In our multivariate analyses, we incorporated age, sex, smoking status, comorbidity, use of NSAID, and type of surgery as potential confounding variables.

Our finding of high age as a potential risk factor for postoperative complications is in agreement with findings from Turrentine et al., examining 7,696 surgical procedures [11]. They concluded that risk factors for postoperative morbidity and mortality increase with age, but they also found high age itself to be an independent risk factor. We could not demonstrate a trend of increasing CCI score with increasing age in our population. This could be explained by the selection of elderly patients. Surgeons are more likely to refer patients in this age group for surgery, if they have few comorbidities and good performance. With regard to 90-day mortality, our results are also in agreement with the results by Turrentine et al., as we could demonstrate an increased 90-day mortality in patients aged $\geq 75$ years. The fact that we could demonstrate an increased 90-day, but not 30-day, mortality in this group stress the importance of reporting the former in all surgical papers. This was also the conclusion by Byrne et al. in a study of 171.688 patients undergoing elective and emergency surgery for colorectal cancer during a six-year period [12]. Further, the International Consensus on Standardization of Data Collection for Complications Associated with Esophagectomy strongly recommended recording the 90-day mortality [4].

The Kaplan-Meier plot reflects the 5-year survival and demonstrate a lower survival rate among patients aged $\geq 75$ years compared with younger patients. This is not a surprise, as some of the fatalities in the highest age group can be attributed to other causes than cancer recurrence. Unfortunately, we were unable to distinguish between disease-free survival and overall survival as we lacked information on causes of death. We present an accumulated 5 -year survival rate of $25 \%$, which is comparable to results from previous reports [13-15].

In our study, the presence of comorbidity (defined as a CCI score $\geq 1$ ) tended to increase the incidence of postoperative complications. In a population-based cohort study on 609 patients undergoing surgery for esophageal cancer, Backemar et al. demonstrated that a CCI score $\geq 2$ was associated with a significantly increased incidence of postoperative complications [16]. Thus, this is in agreement with our findings, although our results were not statistically significant.

Smoking was not associated with adverse outcome following esophageal resections in our study, but we demonstrated that postoperative use of NSAID was strongly associated with an increase in postoperative complications but not mortality. An apparent explanation for this association could be the impairment of NSAID on anastomotic healing, which we have previously examined (submitted). This association with NSAID on anastomotic leakage following gastrointestinal surgery has previously been demonstrated in studies of colorectal surgery $[17,18]$.

Neoadjuvant therapy seemed to protect against postoperative complications with an OR of 0.65 even though there was no statistically significant difference in the distribution of complications across the three age groups. This could probably be explained by patient selection since some patients deemed unfit for neoadjuvant therapy still underwent surgery.

Our leakage rate was $7.6 \%$, which is comparable to previous reports $[19,20]$. Even though there was no difference in distribution of leakage between the age groups, anastomotic leakage was a statistically significant predictor for 90 -day but not 30 -mortality with no fatalities within 30 days and $12.2 \%$ of 
patients with anastomotic leakage dead within 90 days.

All patients included in the present study were resected and reconstructed ad modum Ivor-Lewis. Four hundred seventy-seven patients underwent laparotomy and thoracotomy, 71 laparoscopy and thoracotomy, and only seven patients had a laparoscopy and thoracoscopy performed. The 71 patients, who had a laparoscopy and thoracotomy performed, had a significantly higher complication rate compared with the other patients. Laparoscopy as part of the Ivor-Lewis procedure was introduced in our departments in 2010. In 2013, we examined the results and concluded that the laparoscopic part of the Ivor-Lewis procedure was very complex, and that the learning curve is flat compared to other surgical procedures [21], i. e. this could explain the many complications in this group. In our analyses, we stratified for type of surgery, so this observation did not influence our conclusions.

The present study included consecutive patients undergoing esophageal resection for cancer at two Danish institutions registered in the DECV database. The DECV database is a nationwide, prospectively maintained database covering all Danish patients operated for esophageal cancer since 2003 with a coverage of nearly $100 \%$. Accordingly, this database has high validity. In addition, we obtained information on the comorbid burden from the DNPR, which has registered all admissions to hospitals in Denmark. As a result, our estimates on comorbidity scores are associated with very high validity. Another strength of our study is the incorporation of 90-day mortality, which revealed a higher mortality in the highest age group, not evident from the 30 day mortality estimate.

Some limitations should be kept in mind, when interpreting the results of our study. First, this is a highly selected population. This is emphasized by the lower proportion of smokers, fewer patients treated with neoadjuvant chemoradiotherapy, and fewer open surgeries in the highest age group. Second, we were unable to obtain information on smoking status on $25 \%$ of the patients. As smoking were unequally distributed in the three age groups, this may have confounded our results. Third, our sample in the highest age group is likely to be too small to detect a statistically significant impact of high age on postoperative complications.

In the present study, we chose to examine the effect of age and comorbidity status on postoperative outcomes following esophageal resection. As the incidence of esophageal cancer rises with age, and average life expectancy is increasing, more patients will be diagnosed with esophageal cancer. As resection is the primary curative treatment modality, more elderly patients will be referred to surgery. Thus, knowledge of the expected outcome of esophageal cancer in the elderly is important to make evidence-based clinical decisions as to whom should be offered surgery.

\section{Conclusion}

We found that high age is an independent risk factor for death within 90, but not 30, days after surgery for adenocarcinoma of the esophagus and GEJ. In addition, high age may increase the risk of postoperative complications. The presence of comorbidity may increase the risk of postoperative complications but not death, following surgery for adenocarcinoma of the esophagus and gastroesophageal junction.

\section{Authors' Contributions}

- DWK: Study design, Data analysis \& interpretation, Paper drafting, Critical revisions

- KTF: Study design, Data acquisition, Data analysis \& interpretation, Critical revisions

- JK: Data interpretation, Paper drafting, Critical revisions

- CO: Data acquisition, Data interpretation, Critical revisions

- MPA: Data interpretation, Critical revisions

- LBS: Study design, Data interpretation, Critical revisions

- FVM: Study design, Data interpretation, Paper drafting, Critical revision

\section{References}

[1] Pennathur A, Gibson MK, Jobe BA, Luketich JD: Oesophageal carcinoma. Lancet 2013, 381 (9864): 400-412.

[2] Samalin E, Ychou M: Neoadjuvant therapy for gastroesophageal adenocarcinoma. World journal of clinical oncology 2016, 7 (3): 284-292.

[3] Dindo D, Demartines N, Clavien PA: Classification of surgical complications: a new proposal with evaluation in a cohort of 6336 patients and results of a survey. Annals of surgery 2004, 240 (2): 205-213.

[4] Low DE, Alderson D, Cecconello I, Chang AC, Darling GE, D'Journo XB, Griffin SM, Holscher AH, Hofstetter WL, Jobe BA, Kitagawa Y, Kucharczuk JC, Law SY, Lerut TE, Maynard N, Pera M, Peters JH, Pramesh CS, Reynolds JV, Smithers $\mathrm{BM}$, van Lanschot JJ: International Consensus on Standardization of Data Collection for Complications Associated With Esophagectomy: Esophagectomy Complications Consensus Group (ECCG). Annals of surgery 2015, 262 (2): 286-294.

[5] Lewis I: The surgical treatment of carcinoma of the oesophagus; with special reference to a new operation for growths of the middle third. The British journal of surgery 1946, 34: 18-31.

[6] Charlson ME, Pompei P, Ales KL, MacKenzie CR: A new method of classifying prognostic comorbidity in longitudinal studies: development and validation. Journal of chronic diseases 1987, 40 (5): 373-383.

[7] Backemar L, Lagergren P, Johar A, Lagergren J: Impact of comorbidity on mortality after oesophageal cancer surgery. The British journal of surgery 2015, 102 (9): 1097-1105.

[8] Donders AR, van der Heijden GJ, Stijnen T, Moons KG: Review: a gentle introduction to imputation of missing values. Journal of clinical epidemiology 2006, 59 (10): 1087-1091. 
[9] Sterne JA, White IR, Carlin JB, Spratt M, Royston P, Kenward MG, Wood AM, Carpenter JR: Multiple imputation for missing data in epidemiological and clinical research: potential and pitfalls. Bmj 2009, 338: b2393.

[10] DECV annual report. http://decvgicancerdk/Defaultaspx?pID=17 2014.

[11] Turrentine FE, Wang H, Simpson VB, Jones RS: Surgical risk factors, morbidity, and mortality in elderly patients. Journal of the American College of Surgeons 2006, 203 (6): 865-877.

[12] Byrne BE, Mamidanna R, Vincent CA, Faiz O: Populationbased cohort study comparing 30- and 90-day institutional mortality rates after colorectal surgery. The British journal of surgery 2013, 100 (13): 1810-1817.

[13] Oakley B, Lamb C, Vohra R, Catton J: Achieving long term survival in oesophagectomy patients aged over 75. Annals of medicine and surgery (2012) 2016, 9: 15-21.

[14] Cunningham D, Allum WH, Stenning SP, Thompson JN, Van de Velde CJ, Nicolson M, Scarffe JH, Lofts FJ, Falk SJ, Iveson TJ, Smith DB, Langley RE, Verma M, Weeden S, Chua YJ, Participants MT: Perioperative chemotherapy versus surgery alone for resectable gastroesophageal cancer. The New England journal of medicine 2006, 355 (1) 11-20.

[15] Allum WH, Stenning SP, Bancewicz J, Clark PI, Langley RE: Long-term results of a randomized trial of surgery with or without preoperative chemotherapy in esophageal cancer. Journal of clinical oncology: official journal of the American Society of Clinical Oncology 2009, 27 (30): 5062-5067.
[16] Backemar L, Lagergren P, Djarv T, Johar A, Wikman A, Lagergren J: Comorbidities and Risk of Complications After Surgery for Esophageal Cancer: A Nationwide Cohort Study in Sweden. World journal of surgery 2015, 39 (9): 2282-2288.

[17] [17] Gorissen KJ, Benning D, Berghmans T, Snoeijs MG, Sosef MN, Hulsewe KW, Luyer MD: Risk of anastomotic leakage with non-steroidal anti-inflammatory drugs in colorectal surgery. The British journal of surgery 2012, 99 (5): 721-727.

[18] Klein M, Gogenur I, Rosenberg J: Postoperative use of nonsteroidal anti-inflammatory drugs in patients with anastomotic leakage requiring reoperation after colorectal resection: cohort study based on prospective data. Bmj 2012, 345: e6166.

[19] Escofet X, Manjunath A, Twine C, Havard TJ, Clark GW, Lewis WG: Prevalence and outcome of esophagogastric anastomotic leak after esophagectomy in a UK regional cancer network. Diseases of the esophagus: official journal of the International Society for Diseases of the Esophagus / ISDE 2010, 23 (2): 112-116.

[20] Patil PK, Patel SG, Mistry RC, Deshpande RK, Desai PB: Cancer of the esophagus: esophagogastric anastomotic leak--a retrospective study of predisposing factors. Journal of surgical oncology 1992, 49 (3): 163-167.

[21] Petersen TI, Pahle E, Sommer T, Zilling T: Laparoscopic minimally invasive total gastrectomy with linear stapled oesophagojejunostomy--experience from the first thirty procedures. Anticancer research 2013, 33 (8): 3269-3273. 